Chronic hypoxia regulates Cytoplasmic Polyadenylation Element Binding Protein 2 alternative splicing to promote HIF1a translation

Emily M. Mayo ${ }^{1}$, Shaun C. Stevens ${ }^{1}$, Anika N. Ali ${ }^{1}$, Christina J. Moss ${ }^{1}$, Sean P. Lund ${ }^{1}$, Gina S.

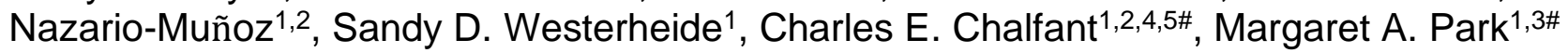

\title{
Author affiliations:
}

${ }^{1}$ University of South Florida, Department of Cell Biology, Microbiology and Molecular Biology

${ }^{2}$ Moffitt Cancer Center, 12902 USF Magnolia Drive, Tampa, FL 33612

${ }^{3}$ Moffitt Cancer Center, Dept. of GI Oncology/Dept. of Bioinformatics and Biostatistics, 12902 USF Magnolia Drive, Tampa, FL 33612

${ }^{4}$ VCU Johnson Center for Critical Care and Pulmonary Research, Richmond, VA 23298

${ }^{5}$ Research Service, James A. Haley Veterans Hospital, Tampa, FL 33612

\#These authors are co-corresponding authors

To whom correspondence should be addressed: Margaret A. Park: Dept. of GI Oncology/Dept. of Biostatistics/Bioinformatics, Moffitt Cancer Center, 12902 USF Magnolia Drive, Tampa, FL 33612; Margaret.park@moffitt.org

Author contributions: Conception and design: EMM, MAP; Performing experiments: EMM, SCS, ANA, CJM, SPL, GSNM, MAP; Analysis and interpretation: EMM, MAP, SDW; Drafting the manuscript: EMM, MAP; Editing manuscript: EMM, MAP, CEC, SDW.

\section{Running Title: CPEB2 controls HIF1 $\alpha$ translation during hypoxia}

\section{GRANT SUPPORT}

This work was supported by research grants from the American Cancer Society (ACS), IRG-17173-22 (MAP), U.S. Department of Veterans Affairs (VA Merit Review, I BX001792 (CEC) and a Research Career Scientist Award, 13F-RCS-002 (CEC); from the National Institutes of Health via TR000057-04 (MAP), AG052149 (SDW), 1U01HD087198-01 (CEC), CA117950 (CEC), CA154314 (CEC) and Al139072 (CEC). The contents of this manuscript do not represent the views of the Department of Veterans Affairs or the United States Government. 


\begin{abstract}
HIF1 (Hypoxia-inducible Factor 1) is a transcription factor that plays a crucial role in the hypoxia stress response. However, chronic hypoxia exposure can cause irreversible physiological changes that can lead to pulmonary hypertension $(\mathrm{PH})$ and the need for therapeutics to ameliorate these conditions is great and unmet. Previous studies in our lab have demonstrated that CPEB2 (cytoplasmic polyadenylation element binding protein 2) is a translational repressor of one of the HIF1 subunits: HIF1a. Our lab demonstrated that the alternatively spliced CPEB2A isoform of CPEB2 is a repressor of translation, while the CPEB2B isoform is a translational activator of HIF1a during hypoxia, suggesting a major regulatory role for CPEB2 AS in the pulmonary hypoxic response. Although it is well established that during hypoxia, HIF1a levels are dramatically upregulated due to a decrease in the degradation of this factor, we propose that during chronic hypoxia, the expression of HIF1a is maintained via a translational mechanism, likely alongside a decrease in proteolytic degradation. In this study we demonstrate that depletion of the CPEB2B splice isoform has an inhibitory effect on the translation of nascent HIF1a protein during chronic hypoxia, but not the acute phase. We further demonstrate this pathway is dependent on the initiation factor elF3H. Finally, we show data which indicate CPEB2A and CPEB2B bind differentially to cytoplasmic polyadenylation element consensus sequences depending on surrounding sequence context. These findings are important since they provide evidence for the potential of CPEB2 to act as a therapeutic target for treating chronic hypoxia-related pulmonary diseases.
\end{abstract}

Keywords: chronic hypoxia, alternative splicing, CPEB2, HIF1

\title{
INTRODUCTION
}

When hypoxic conditions are encountered, the human pulmonary system undergoes alterations in gene and protein expression in order to adapt to an oxygen deprived environment. Under the control of hypoxia-inducible factors (HIF proteins), the transient expression of angiogenic and vasoconstrictive genes can help attenuate the hypoxic stress response. However, over longer exposures, expression of HIF-dependent genes can lead to detrimental and irreversible effects such as rearrangement of the lung vasculature and eventually occlusion of blood vessels $(\mathbf{1}, \mathbf{2})$ As an example, one of the most common causes of chronic hypoxia $(\mathrm{CH})$ in the lungs is chronic obstructive pulmonary disease (COPD), commonly induced by smoking and accounting for as many as 8 out of 10 COPD-related deaths (3). As a more timely example, COVID-19 patients often demonstrate hypoxia secondary to their infection in the lungs (4). In addition to COPD and COVID-19-mediated hypoxia, the chronic hypoxic response can be initiated by various pulmonary cancers, acute lung injury, sleep apnea, obesity and smoking. Treatment for these conditions has been limited to vasodilators and oral medications which, alongside hospital stays that cost around $\$ 110$ billion US dollars annually to treat $(3,5)$.

To compensate for the poor oxygen flow throughout the lungs of a patient with chronic hypoxia, endothelial and smooth muscle cells throughout the pulmonary system proliferate, blood vessels constrict, and pulmonary blood pressure rises. These pathological, yet at first necessary changes ultimately lead to pulmonary hypertension and in the most severe cases, right-ventricular heart failure and death $(6,7,8)$. Targeting symptoms at the source could potentially alleviate this cost and the morbidity associated with $\mathrm{CH}$ and it is thus important to understand how the $\mathrm{CH}$ response mechanism is regulated. To address gaps in current 
knowledge, our study aims to identify novel regulators of the hypoxic response with a focus on the chronic phase of the response, to open the door to new therapeutic targets.

Under normoxic conditions, the constitutively expressed HIF1 $\beta$ subunit remains in the cytosol, whereas a shift into hypoxia is required to increase expression of the short-lived HIF $1 \alpha$ subunit (9). The current reigning dogma for HIF1a expression is that during hypoxia, HIF1a levels increase due to a decrease in HIF1a ubiquitination. When oxygen is present, HIF1a is hydroxylated on conserved proline residues $(9,10)$ causing its recognition by the von Hippel Lindau (VHL) ligase (5,9). VHL tags HIF1a with ubiquitin, targeting it to the proteasome for degradation. However, as oxygen levels drop, HIF1 $\alpha$ accumulates and binds to its partner, HIF1 $\beta$, forming the HIF1 complex. This complex acts as a transcription factor to downstream targets (11-13).

One such target of HIF1 is Vascular Endothelial Growth Factor (VEGF). Transcription of VEGF is regulated by the presence of a hypoxia response element (HRE) within the promoter of the VEGF transcript, which allows HIF-1 to both bind to the VEGF promoter and induces its expression during low oxygen conditions. Once transcribed, the binding of VEGF to its receptor (VEGFR) induces angiogenesis and promotes vasculogenesis (14, 27,30-35).

Although the ubiquitination/proteasomal degradation mechanism of HIF1a regulation is well established, our lab has observed prolonged HIF1 a expression during the chronic phase of hypoxic stress, indicating a potential secondary mechanism that maintains HIF1a expression levels during chronic hypoxia. Published studies in our lab have demonstrated that the alternative splicing (A/S) of cytoplasmic polyadenylation element binding protein 2 (CPEB2) may play a role in HIF1a expression during the chronic hypoxic response (15). CPEBs are a family of proteins (CPEB1, CPEB2, CPEB3, and CPEB4) that generally act to suppress mRNA translation by modulating polyadenylation $(15,16)$. Based on published work from our lab, the $A$ and $B$ isoforms of CPEB2 differ by a single 90 base-pair exon (15). This exon is excluded in the CPEB2A isoform, while it is included in the CPEB2B isoform. A splicing factor known as SRSF3 (arginine/serine-rich splicing factor 3$)(15,17,31)$ binds to a consensus sequence near the $3^{\prime}$ end of the exon, forcing its inclusion in the final transcript of CPEB2B $(15,17)$. In this manuscript, we provide evidence that the $B$ isoform of CPEB2 is required for the translation of new HIF1a protein specifically during the chronic stages of hypoxia exposure in vitro. Furthermore, we provide data indicating that the elF3H subunit of the pre-initiation complex may aid CPEB2B in initiating HIF1 $\alpha$ translation via preferential association with this protein.

Therefore, the alternative splicing of CPEB2 is a novel regulator of HIF1 a during hypoxia. Future studies may be possible to target the expression of CPEB2B in COPD and other hypoxialinked animal models and eventually aim to regulate HIF1 in patients suffering from chronic hypoxic disorders.

\section{RESULTS}

CPEB2 alternative splicing is dysregulated during chronic hypoxia. Seminal studies in the field have demonstrated that CPEB2 localizes via a low complexity sequence to stress granules which also contain mRNA transcripts (32). Generally, binding occurs at consensus sequences called cytoplasmic polyadenylation elements (CPE sites) $(\mathbf{1 8 , 1 9 )}$. These findings indicate that members of the CPEB family play a large role in the response to stress, as the mRNA species found in stress granules are linked to stress processes (15-19). In light of the current literature and our previous findings, we assessed whether exposure to hypoxia would alter the CPEB2 $\mathrm{A} / \mathrm{B}$ expression levels. For the purposes of these studies in a cell model, we define the acute 
hypoxic response as occurring prior to 24 hours and chronic exposure from 24-96 hours (the longest we have treated)(20). In general agreement with others' findings, we found that HIF1a protein levels increased as early as 4 hours after exposure to hypoxic conditions in vitro $\left(2 \% \mathrm{O}_{2}\right)$ and remained elevated well into 96 hours of constant exposure to hypoxic conditions. Correlative to these findings, the ratio of CPEB2A to CPEB2B dramatically decreased during later time points (Fig. 1A). Hence, we observed a reliable decrease in the CPEB2 A/B ratio as hypoxia progresses into the chronic stage (Fig. 1A, S1). Primary pulmonary arterial endothelial cells (hPAECs) demonstrated a similar pattern in both HIF1 $\alpha$ protein and the CPEB2A/B ratio over the course of 96 hours (Fig. 1B,F,G). A Pearson's correlation analysis demonstrates a negative correlation between CPEB2A/B ratio and HIF1a levels (correlation coefficient of -0.72; Fig. 1CE).

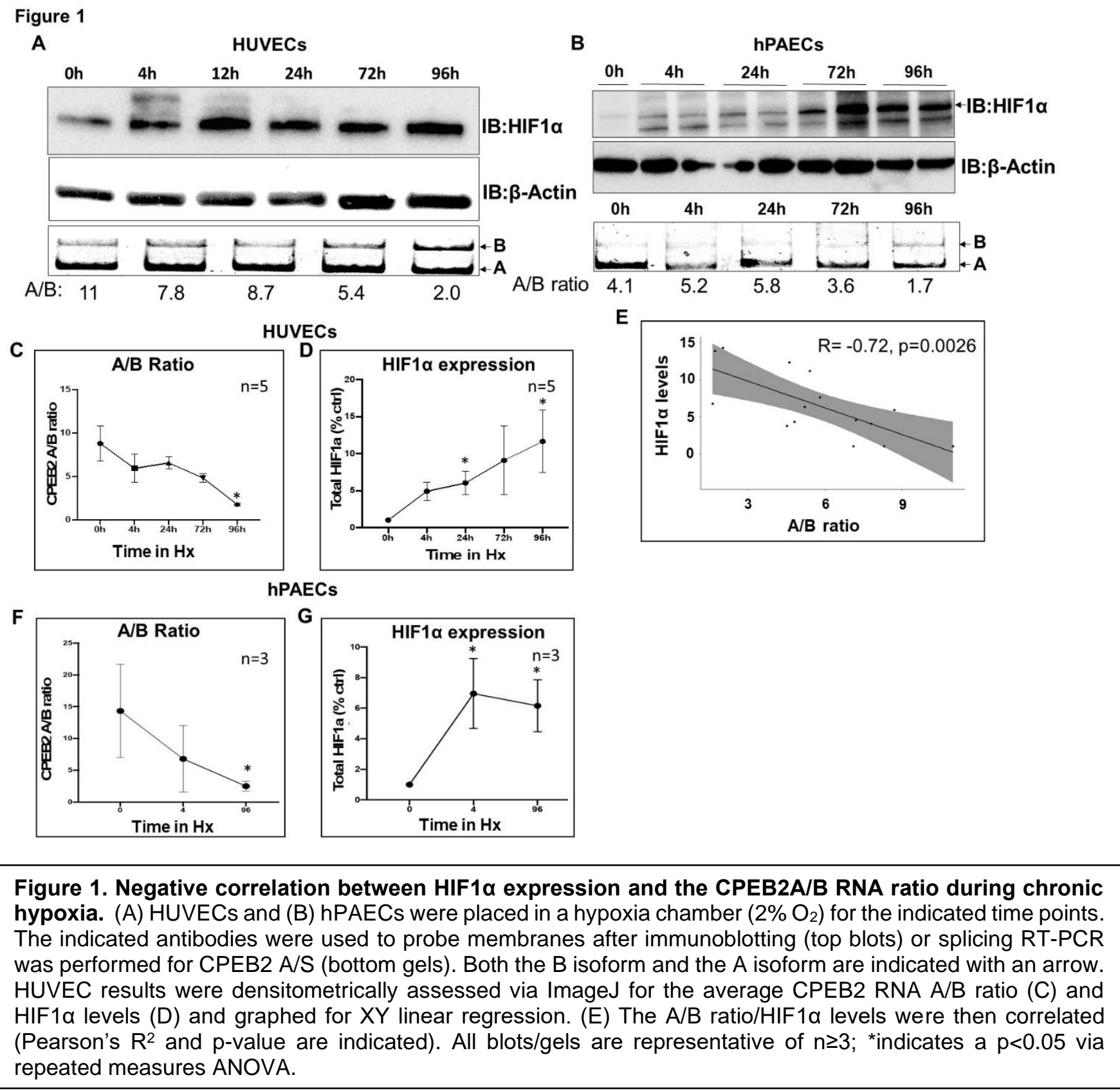




\section{Figure 2}

A

CPEB2 mRNA transcript: Included exon (CPEB2B)

AUAGUUGGUGCACUGCAGCCGGAACAUCCAGAAUAGACCAG

GGCCUUGUAGGUCUUAUCUGGUC CPEB2BASO

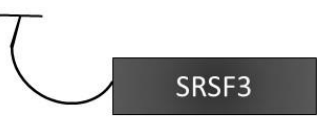

B

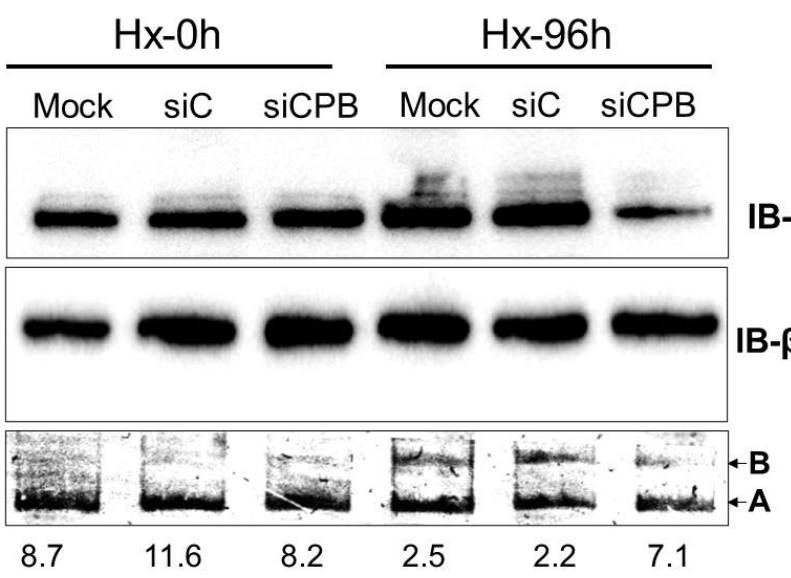

IB-HIF1a

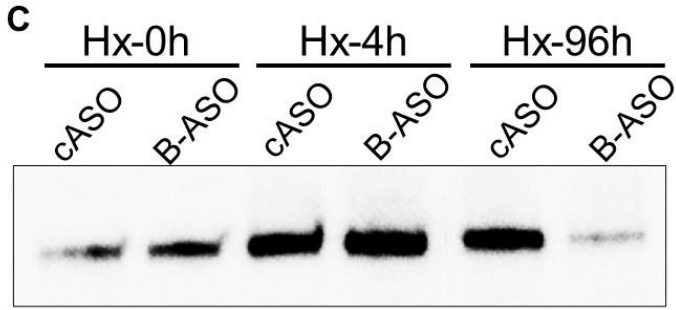

A/B:
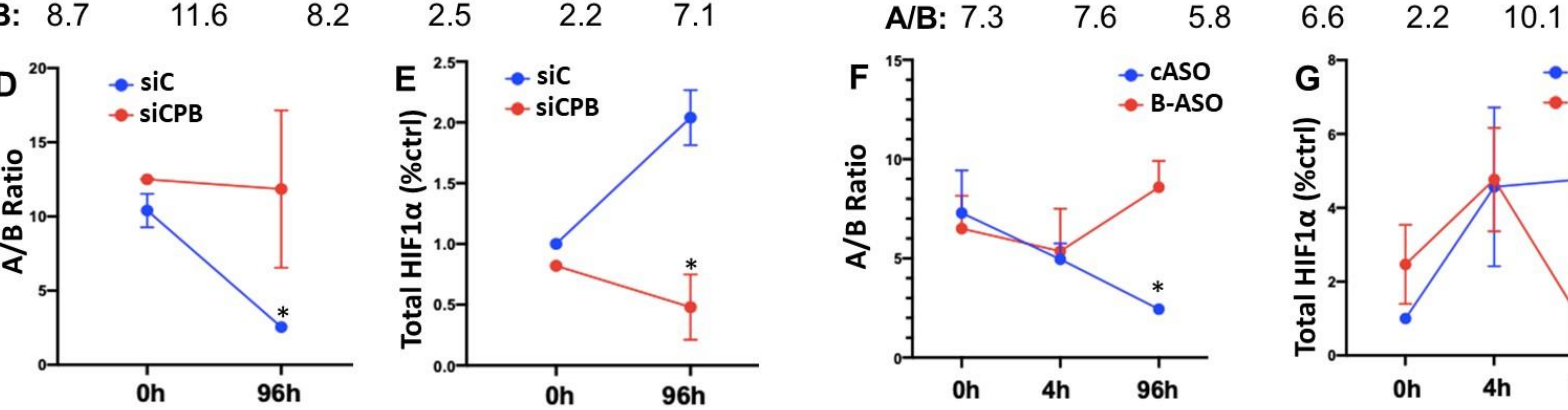

H CASO: $\mathrm{Hx}$ Oh
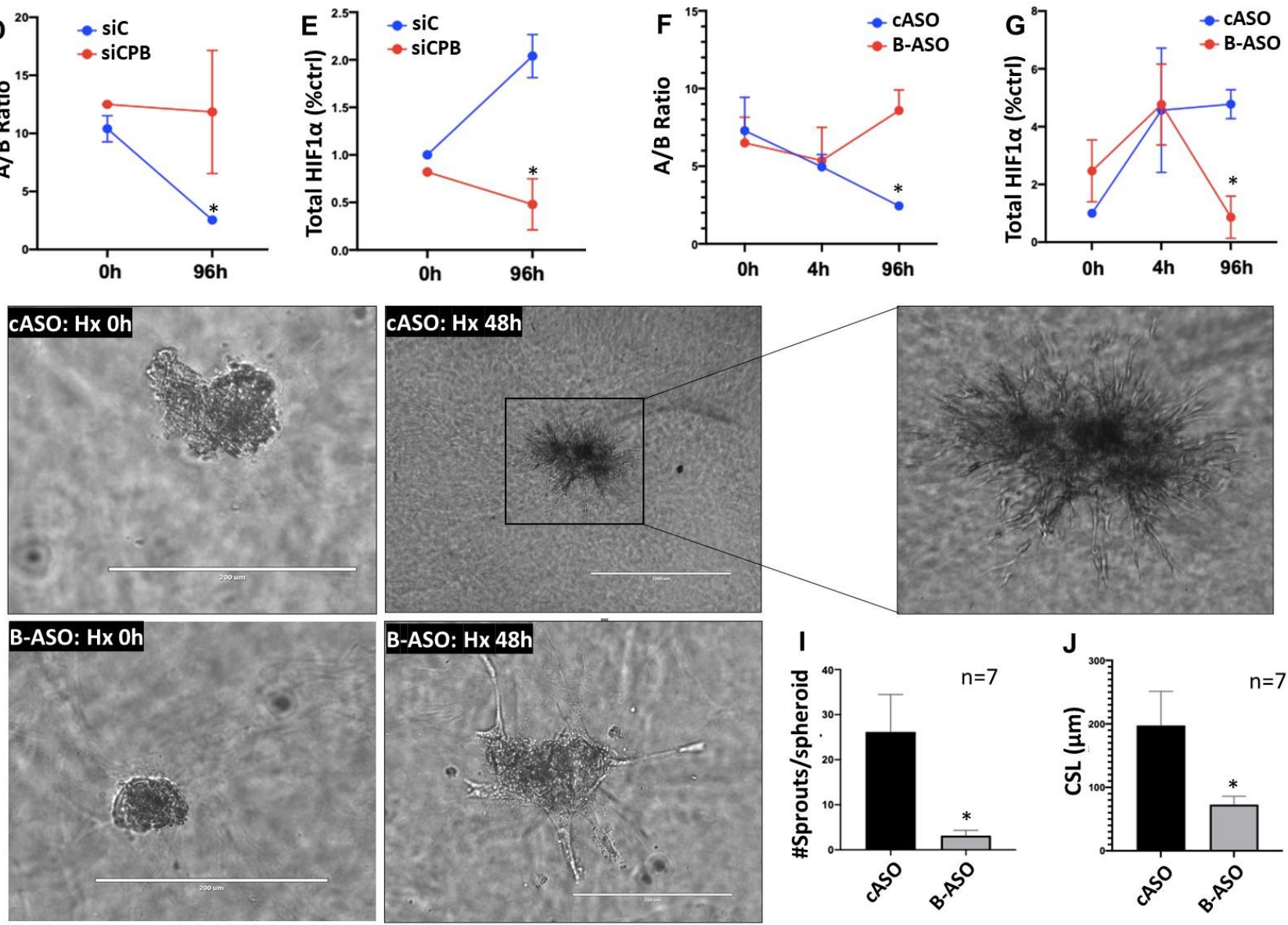
Figure 2. CPEB2B regulates HIF1 $\alpha$ expression during Chronic Hypoxia. A) Schematic of the CPEB2B ASO design. (B-C) HUVECs (N=3: P3, P4) were subjected to hypoxia $\left(2 \% \mathrm{O}_{2}\right)$ for the indicated times $-/+$ either mock conditions (Mock), control siRNA (siC) or siRNA targeted towards CPEB2B (siCPB), (B); or control ASO (cASO) or CPEB2B ASO (B-ASO) (pre-treated, C). Both RNA and protein were harvested and subjected to either immunoblot using the indicated antibodies (top blots) or was subjected to quantitative "splicing" RT-PCR for CPEB2 A/S (bottom gels). (D-G) Change in the CPEB2 A/B ratio (D,F) and HIF1a protein expression (E,G) were assessed via densitometry for depletion of CPEB2B either via siRNA $(D, E)$ or ASO $(F, G)$. H-J) HUVEC spheroids were assessed via microscopy $(\mathrm{H})$ and both number of sprouts per spheroid (I) and cumulative sprout length (CSL, J) were measured. The scale bar for all micrographs is 200 microns except for the control ASO spheroid at $48 \mathrm{~h}$ (the spheroids grow larger during sprouting, inset is a blown-up image to show sprout number). ${ }^{*}$ indicates $p<0.05$ via repeated measures ANOVA or t-test.

\section{CPEB2B regulates HIF1a protein levels during chronic hypoxia.}

As previously demonstrated by our lab ectopic expression of CPEB2A leads to a decrease in HIF1a protein expression, whereas ectopic expression of CPEB2B will lead to an opposing increase of this factor during normoxic conditions (15). Expanding on these findings, we assessed the impact of CPEB2B depletion on the expression of HIF1 $\alpha$ during both acute and chronic hypoxia phases.

Our lab has established that the alternative splicing of CPEB2A is at least partially regulated by the trans-factor SRSF3 (Serine/Arginine-rich splicing factor 3) (15-17). Therefore, we used antisense oligonucleotides (ASOs) to block the binding site of SRSF3 to its consensus sequence in exon 4 of CPEB2B (Fig.2A). If SRSF3 cannot bind to its consensus sequence, exon 4 will be excluded from the final transcript and the ratio of CPEB2A/B will increase as shown in Fig. 2C, 2F. CPEB2B knockdown mediated by both antisense oligonucleotides (Fig.2C) and siRNA (Fig. 2B) targeted towards CPEB2B (included exon) confirmed that HIF1a expression is in part dependent on CPEB2B expression (Fig. 2B-C) during the chronic phase of hypoxia. More specifically, we find that inhibition of CPEB2B at the later 72-96 $\mathrm{h}$ time point prevented HIF1a expression leading to expression values similar to those observed under normoxic conditions (Fig. 2B-E).

\section{HIF1a is synthesized at the translational level during Chronic Hypoxia.}

A large body of published data demonstrates that increases in HIF1a during hypoxia are due to a decrease in ubiquitination of this protein. However, published work in our lab has demonstrated that CPEB2 A/S plays a regulatory role in the translation of HIF1a (15). Hence, it is plausible that CPEB2 A/S allows the maintenance of enhanced HIF1a expression levels during later hours of hypoxia via nascent protein synthesis. Indeed, we find that inhibiting protein synthesis via cycloheximide induced a shift in HIF1a expression both in HUVEC's and in hPAEC's. After 4 hours of exposure, inhibition of translation had no significant effect on HIF1a expression, compared to 96 hours of exposure, where HIF1a protein levels were comparable to those observed during normoxia (Fig. 3A,B; S2). VEGF expression is also decreased at this time point after cycloheximide, both in HUVECs and hPAECs (data not shown). Interestingly, we find that cycloheximide induces HIF1a expression under normoxic conditions (Fig.3A, S2), perhaps due to the induction of translational stress, and that our data at 4 hours of hypoxia are somewhat variable. Hence, we assessed nascent protein production during short and long-term hypoxia exposure to confirm our findings.

\section{CPEB2 alternative splicing regulates HIF1a translation during chronic hypoxia.}

In general agreement with our data in Fig. 3 A,B, we used "CLICK" chemistry to detect nascent HIF1a protein via incubation with the methionine mimic with L-azido-homoalanine (AHA) and subsequent labeling with biotin (Fig. 3C). Nascent HIF1a protein was only observed at the 96 
hour time point, but not the acute 4 hour time point. Nascent HIF1a expression was inhibited by the CPEB2B ASO, indicating that this pathway is dependent upon CPEB2B expression (Fig. 3D-E).

\section{CPEB2B induces downstream VEGF expression.}

When hypoxic conditions are encountered, the HIF1 transcription factor binds to hypoxic response elements within the promoter of transcriptional targets such VEGF (Fig. 4A). VEGF then induces vascular formation at the site of hypoxia. Therefore, we wished to establish whether targeting CPEB2 A/S can regulate downstream HIF1a targets linked to angiogenesis such as VEGF and thus we inhibited CPEB2B expression using B-ASO. We found that knocking down CPEB2B attenuates the expression of downstream targets of HIF1a, such as VEGF.

Figure 3. HIF1 $\alpha$ levels are maintained by translation during chronic hypoxia. (A-C) HUVECS (P3-P6) and hPAECs $(P=5)$ were incubated with cycloheximide $(5 \mathrm{ug} / \mathrm{ml}) 4$ hours prior to harvesting and blotted with the indicated antibodies. HIF1a levels were assessed via ImageJ and graphed for total protein. (D-E) Nascent proteins were labelled with biotin, then precipitated using streptavidin (PD:Biotin). Labelled proteins were immunoblotted (IB) using the indicated antibodies. Blots in (E) and replicates in (D) represent biological replicates. Blots are representative of an $n \geq 3 ;{ }^{*}=p<0.05$ via repeated measures ANOVA.

\section{Figure 3 hPAEC}

A

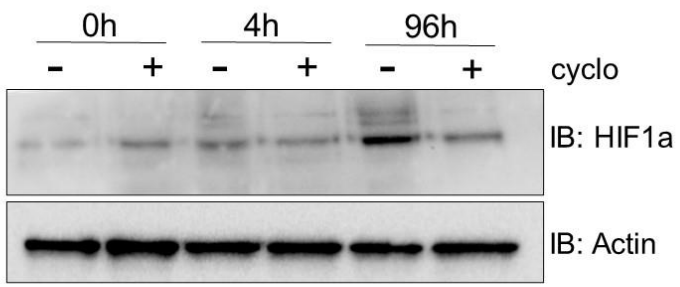

HUVEC
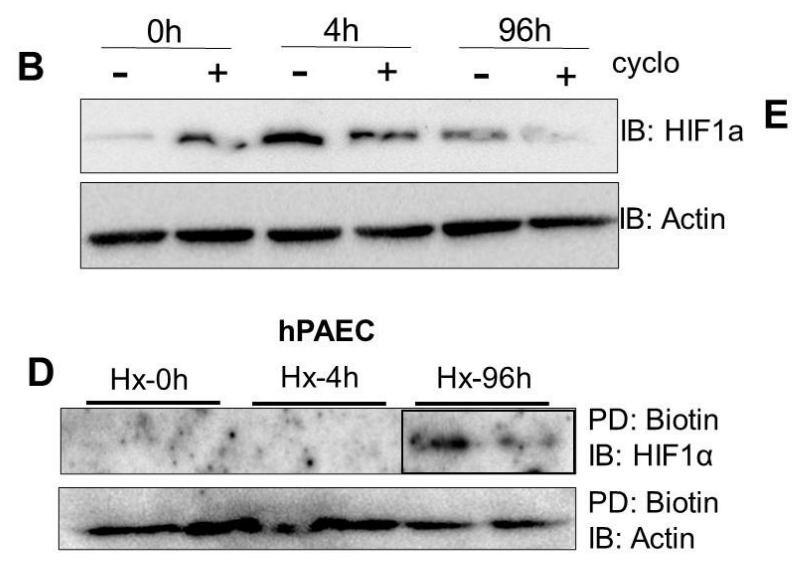

C
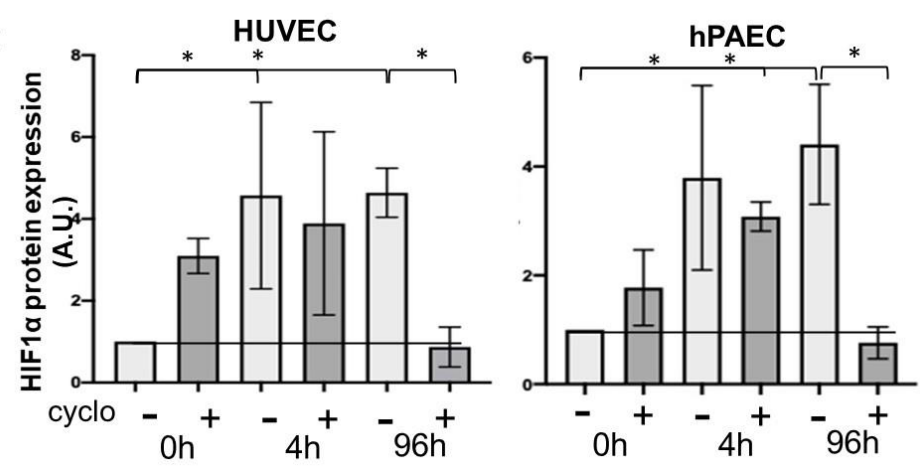

HUVEC

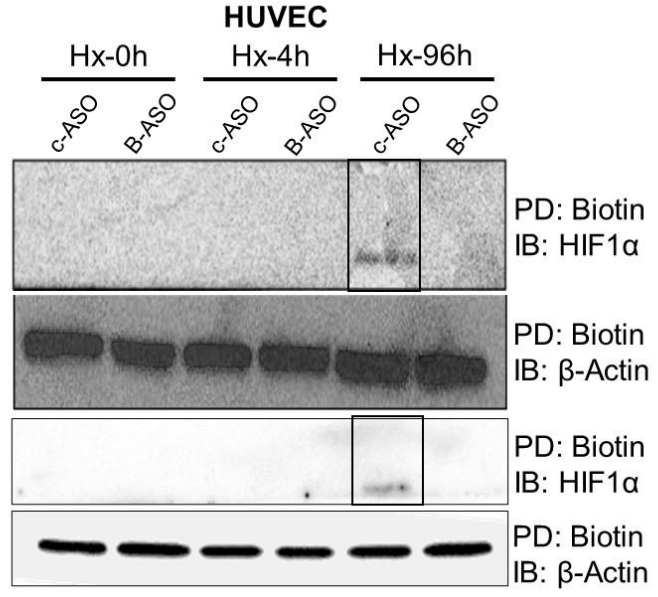


Figure 4. CPEB2B regulates downstream VEGF expression during chronic hypoxia. $(A)$ Representation of HIF1 $\alpha$ activity. (B-C) HUVECs were transfected with the indicated ASOs (control or CPEB2B) or a plasmid to express HIF1a, then exposed to hypoxia. Immunoblots were then performed with the indicated antibodies (repeat blots represent biological replicates). Densitometry was determined (shown in $C$ ). $n \geq 3$ for all assays;

Figure 4

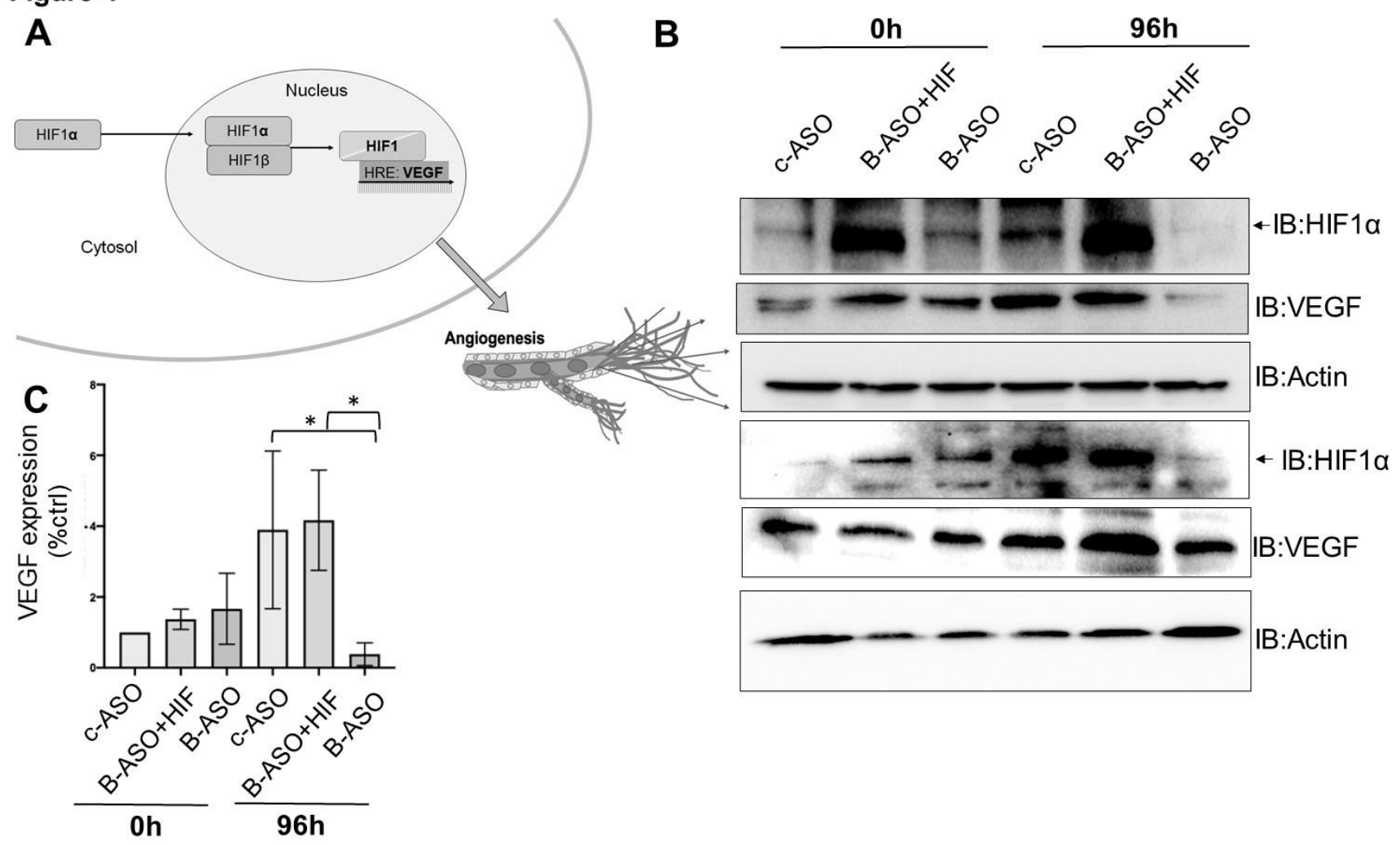

Interestingly, forced expression of HIF1a "rescues" this effect indicating that HIF1a may lie downstream of CPEB2B in the pathway (Fig. 4B-C).

CPEB2B binds differentially to MRNA sequences and regulates HIF1a translation at least partially via elF3H.

To ascertain why CPEB2B function varies from the CPEB2A isoform, we assessed the RNA binding capabilities of the CPEB2 isoforms. Our results indicate that CPEB2A binds to RNA sequences in the HIF1a 3'UTR directly as demonstrated by a gel shift assay (Fig.5A-B, S4). We then wished to determine if CPEB2B binds differentially to this same site. We therefore used biotinylated RNA as "bait" to pull down immunoprecipitated CPEB2A or CPEB2B. Our findings indicate that CPEB2A binds robustly to sequences surrounding the CPE sites in the 3'UTR of both HIF1 $\alpha$ and TWIST1, another RNA species with CPE sites in its 3'UTR. CPEB2B on the other hand, does not bind to the HIF1a CPE site as readily as CPEB2A (Fig.5A,C, S4). Further analysis of the HIF1a mRNA sequence indicates that there are other CPE consensus sequences in the coding region, specifically in exon 10 of the mRNA sequence. We therefore used biotinylated sequences surrounding this sequence in binding assays using immunoprecipitated CPEB2A and B (see Table 1). We find that, while the A isoform binds more readily to the 3'UTR sequence, the $B$ isoform binds preferentially to the sequence in exon 10 of HIF $1 \alpha$, indicating that inclusion of exon 4 in the CPEB2 protein may shift sequence specificity of this factor (Fig. 5D). 
We have demonstrated in Figures 1-3 that HIF1a translation is controlled at least partially via CPEB2 alternative splicing. We therefore wished to identify additional components of this pathway. Hutt et al (21) have demonstrated that HIF1a translation may be regulated by initiation factor elF3H. We therefore wished to determine if CPEB2B may be acting via this mechanism. We demonstrate in Fig. 5E-F that not only does inhibition of elF3H via RNAi decrease CPEB2Bmediated HIF1 $\alpha$ expression, but that this mechanism is likely due to translation as demonstrated in Fig. 5F, S4.

Figure 5

A

HIF1 $\alpha$ 3'UTR

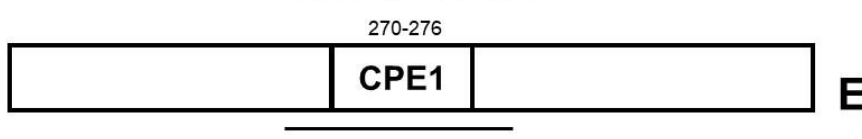

E

B
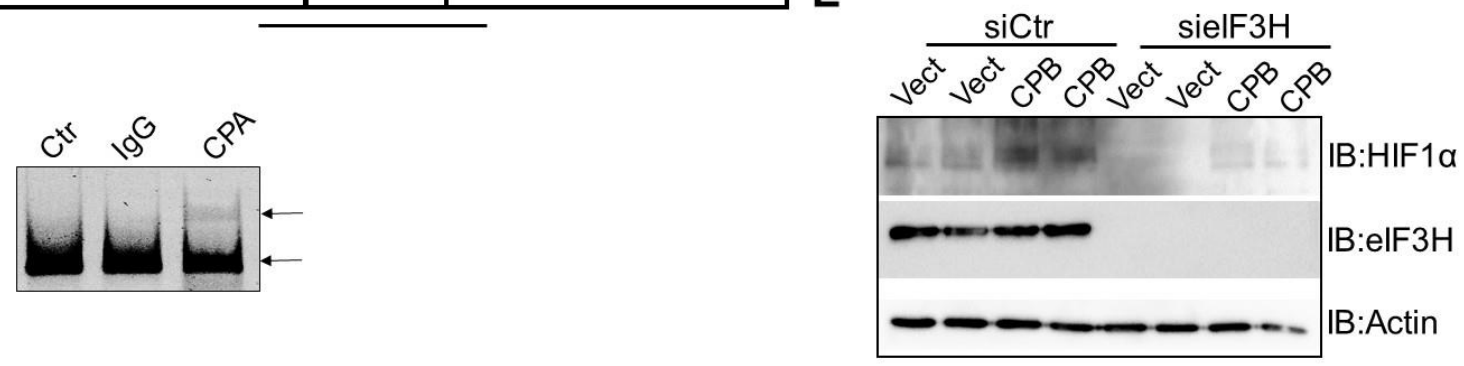

C

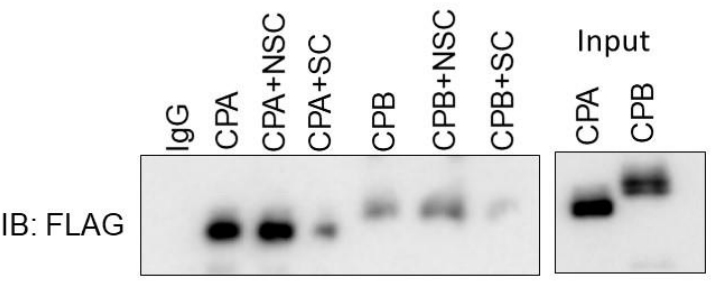

$\mathbf{F}$

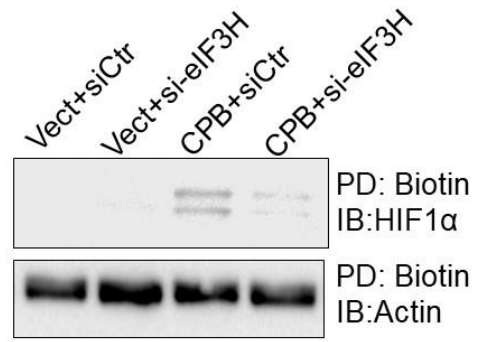

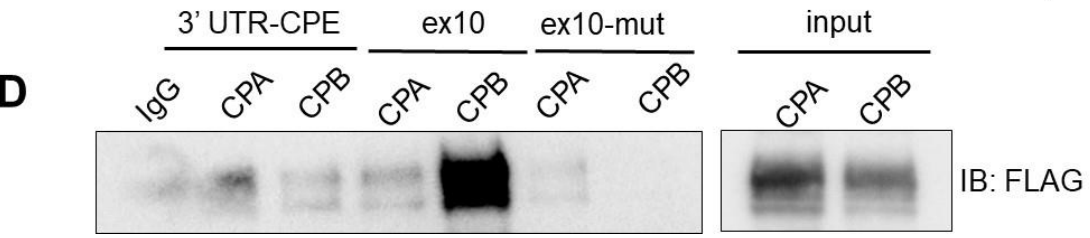

Figure 5. CPEB2B binds differentially to the 3'UTR region and to a region of HIF1 $\alpha$ exon 10 and regulates HIF1a expression via elF3H. (A) Schematic representation of the HIF1a 3' UTR (CPE=cytoplasmic polyadenylation element, $\mathrm{pA}=$ alternative polyadenylation site). (B) FLAG-tagged CPEB2A was immunoprecipitated and exposed to FITC-labeled RNA sequences surrounding the HIF1a 3' UTR CPE sites (CPE). Electrophoretic shift assay was performed. (C) Streptavidin-biotin affinity pull-down was performed using immuno-purified CPEB2A or CPEB2B with sequences corresponding to the 3' UTR of HIF1a or TWIST1 published CPE sites (B) NSC=non-specific competitor, $\mathrm{SC}=$ specific competitor. (D) Streptavidin biotin affinity pulldown using either the 3'UTR CPE site, or sequences surrounding the CPE site in HIF1 $\alpha$ exon 10. (E-F) HUVECs were transfected with published siRNA targeting elF3H. Some samples were subjected to nascent protein labeling $(F)$, then immunoblotted using the indicated antibodies $(E-F)$. $n \geq 3$ for all assays.

\section{DISCUSSION}

Structural changes to the pulmonary vasculature as a result of chronic hypoxia can lead to elevated and irreversible increases in arterial pressure and downstream pathologies. In spite of current treatment methods, pulmonary hypertension remains a prevalent condition 
characterized by thick arterial walls, high blood pressure, and shortened survival. By targeting the genes involved in the hypoxic response, the integrity of the arterial walls might be preserved, and disease progression alleviated. Consequently, understanding and characterizing the mechanisms behind this response could benefit the development of future therapeutics involved in hypoxia-induced pathologies.

It is well documented that CPEB family members bind to specific mRNA targets, thereby preventing translation (19). We find that the CPEB2B isoform has the opposite effect for some targets, inducing the translation of HIF1 $\alpha$ and TWIST1 $(15,16)$. Hence, the inclusion of the small 30 amino acid exon in the CPEB2B sequence dramatically changes the function of this protein $(\mathbf{1 5}, \mathbf{1 6})$. In both these and previous studies, we find that, in contrast to the function of the $A$ isoform, expression of HIF1a during the hypoxic response is sustained at least in part by translation via the CPEB2B isoform. Previous findings from our laboratory indicate that the role of CPEB2B in cancer metastasis is key to the advancement of disease in triple negative breast cancer and that this increase in hypoxia-linked factors is due to translation (14-16). We hypothesize that the Furthermore, the presence of the CPEB2B isoform is critical for nascent HIF1a protein production at later stage timepoints during oxygen deprivation and this is further supported by our data demonstrating that VEGF expression may be regulated via CPEB2 A/S. Our hypothesis to explain this phenomenon is that there is a transitional stage between acute and chronic phases of hypoxia and that shift leads to increased HIF1a translation alongside of decreased degradation. One interesting finding is that HIF1 $\alpha$ protein expression is increased basally after cycloheximide treatment. Hence, we hypothesize that the cycloheximide itself induces a small amount of stress. Indeed, it has been demonstrated that cycloheximide can deplete ubiquitin in cell systems (36). It is therefore plausible that this depletion affects HIF1a levels basally.

With respect to our HUVEC spheroid assay, both sprout length and number were dramatically decreased after hypoxia when CPEB2B is inhibited. However, we did note that, when treated with CPEB2B ASO, sprout diameter seemed to be increased. Although we cannot say for certain why the CEB2B ASO increased sprout diameter, we hypothesize that a physical explanation is possible. It may be that the large number of sprouts induced by hypoxia in our control spheroids made it such that there was no physical room for larger diameter sprouts. Furthermore, the spheroids derived from HUVECs treated with CPEB2B ASO did not grow in culture at the same rate as control and were, therefore, smaller as shown in Figure 2 . This observation is in general agreement with our findings in cancer models that CPEB2B over, in contrast to the A isoform, greatly increases tumor size and metastasis (15-16).

Regarding the mechanism of function of CPEB2B, there are multiple possibilities for why it is different than that of CPEB2A. We find that the proteins seem to bind differentially to UUUUUAU consensus sequences based on the surrounding sequence (based on our findings in Fig.5). This finding is suggestive of a possible shift in the RNA binding domain to bind more readily to other sequences surrounding the consensus CPE site. Analysis of the binding domain of CPEBs by Richter and colleagues suggests that both RNA recognition motifs as well, as the $\mathrm{N}$-terminal zinc finger domain are required for binding (22). Furthermore, RNA footprinting studies by this group have elegantly demonstrated that the secondary structure of RNA is important for binding of CPEB family members (23). Yet others have demonstrated a "fly-trap" mechanism of RNA binding whereby the two RRM domains enclose the RNA sequence (24). other sequences that are important for CPE recognition include the polyadenylation sequences. However, we do not observe a polyadenylation consensus sequence in proximity to the exon 10 
site. Hence, we hypothesize that changes in the $\mathrm{N}$-terminal domain causes changes in the secondary structure of the RNA which is recognized by CPEB2. The global sequences chosen for binding by CPEB2 isoforms is an active topic of research in our laboratory.

Regarding exon 4 of CPEB2, the structure of RNA-bound CPEB2B RRMs is currently unknown. However, IUPRED and Anchor analysis of the sequence of CPEB2A and CPEB2B indicates that the included exon is likely a protein-protein interaction domain and is a slightly intrinsically disordered to slightly ordered region (Fig.S3). Hence, it is possible that CPEB2B targets bound mRNA species to the translational machinery via protein-protein interactions. Chen et al. (25) have identified an eEF2 interacting domain N-terminal to the included exon for

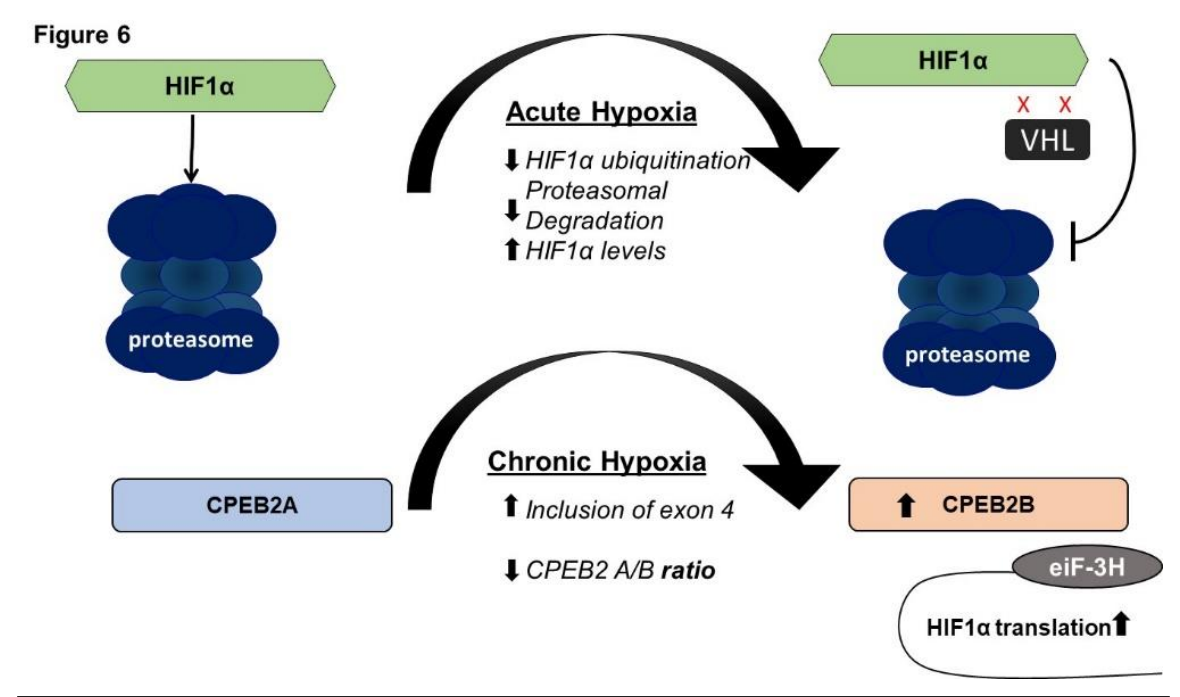

Figure 6: Schematic representation of CPEB2B-mediated HIF1a translation during chronic hypoxia

CPEB2B, which is responsible for CPEB2A-mediated inhibition of expression of HIF1a. It is thus possible that CPEB2B binds to other factors which then mediate elF3Hdependent translation of mRNAs and we will pursue this possibility in future studies.

In conclusion, we have demonstrated that a CPEB2Bmediated translational mechanism may also play an important role during the chronic phase of the hypoxic response pathway. Our data indicate that CPEB2B may cooperate with elF3H to induce translation of the HIF1 a mRNA during the chronic phase of the cellular hypoxic response. However, it is not known precisely how this happens. Thus, future directions for this research include animal studies and studies to determine the factors that direct sequence and secondary structure choice when binding to RNA. HIF1 signaling plays a major role in the pulmonary response to chronic hypoxia. Therefore, patients with adverse pulmonary outcomes secondary to the chronic hypoxic state may benefit from these findings after future studies have been completed.

\section{MATERIAL AND METHODS}

Cell culture. All cell lines (HUVEC (CRL-1730), hPAEC (PCS-100-022), HEK293 (CRL-1573)) were purchased from ATCC (American Type Culture Collection) and cultured according to manufactures instructions. Both endothelial cell lines (HUVEC, hPAEC) were grown in EBM-2 media with EGM-2 bullet kit (purchased from Lonza). No additional serum was used for cell culture. HEK393 cells were grown in DMEM (10\% FBS, 5\% P/S). All experiments were performed on HUVEC's and hPAEC's ranging from passage 1 to passage 6 . When needed, cells were split with Trypsin-EDTA (0.25\%). 
Antibodies and reagents. Two different HIF1a (105SS and 610958) antibodies were used from Novus Biotechnologies and BD Transduction Laboratories. Anti-VEGF (MA513182) was purchased from ThermoFisher. Cycloheximide (C7698) was purchased from Sigma-Aldrich. Click-it protein reaction buffer kit was purchased from Thermofisher. Biotin for click it reaction was purchased from Sigma. All primers and RNA sequences used in this study were purchased from Integrated DNA Technologies (IDT). The HA-HIF1alpha-pcDNA3 plasmid was a kind gift from Dr. William Kaelin (Addgene plasmid \#18949).

Hypoxia exposure. A Modular Incubator Chamber (MIC-1) was purchased from BillupsRothenberg Inc. Custom hypoxia mix ( $2 \%$ oxygen, $5 \%$ carbon dioxide, balance nitrogen, size 200 certified standard-spec cga 580) was purchased from Airgas USA LLC. Cells plated on either $10 \mathrm{~cm}$-dishes or 6-well plates (Corning, Sigma Aldrich) were placed in the chamber with humidified air. Hypoxic air was flushed into the tank for 3-5 minutes, sealed, and added to a $37^{\circ} \mathrm{C}$ incubator for 10-15 minutes before re-administering hypoxic air for another 3-5 minutes. Cells were left in the chamber and incubated at $37^{\circ} \mathrm{C}$ for indicated times.

\section{Antisense oligonucleotide and plasmid transfection.}

ASOs and plasmids were both transfected using Lipofectamine 2000 reagent (11668019) purchased from Thermo-Fisher. Each transfection master mix was incubated in Opti-MEM for 20 minutes prior to adding to serum-starved cells. Cells were then incubated [overnight for plasmid transfection and co-transfection, 30 hours for ASO transfection] before replacing media with complete medium.

Quantitative "Splicing" RT-PCR. cDNA was synthesized using the Superscript III kit (Life Technologies) according to the manufacturers' instructions. cDNA libraries were subjected to traditional PCR as described previously $(16,26,27)$ using primers located on either side of exon 4 of the CPEB2 gene as described previously (16). Ratios were then calculated using densitometry/ImageJ as described by us previously (15-17).

Immunoblotting. Total protein $(15-30 \mu \mathrm{g})$ was electrophoretically separated on $10 \%$ polyacrylamide gels. Samples were transferred electrophoretically to PVDF membranes, then probed with the appropriate antibody as described previously $(\mathbf{1 6}, \mathbf{2 6}, \mathbf{2 7})$.

Immunoprecipitation. Indicated proteins were immunoprecipitated as described previously using anti-FLAG antibodies (15-17).

Streptavidin-biotin affinity pull-down assay. Protocol was adapted from Chen et al, 2012 (25). Biotinylated RNA HIF1 $\alpha$ 3' UTR sequences surrounding the CPE site were purchased from IDT Cells transfected with CPEB2A-flag tagged or CPEB2B-flag tagged were lysed via freezethaw in binding buffer. FLAG-tagged CPEB2 isoforms were immunoprecipitated as described previously $(15,16)$ then incubated with biotinylated RNA sequences in binding buffer $(100 \mathrm{mM}$ $\mathrm{KCL}, 10 \mathrm{mM}$ HEPES, $0.1 \mathrm{mM} \mathrm{CaCl} 2,1 \mathrm{mM} \mathrm{MgCl}_{2}, 5 \%$ glycerol, $100 \mathrm{uM} \mathrm{ZnCl}_{2}, 0.1 \mathrm{mg} / \mathrm{mL} \mathrm{BSA}$, and $1 \times$ protease phosphate cocktail and $1 \times$ RNAse out, plus $5 \mathrm{mM}$ DTT and tRNA). Samples were incubated at room temperature for 30 mins and allowed to rotate overnight with streptavidin beads (washed in binding buffer). Beads were then washed in 1x binding buffer three times and proteins were eluted Laemmli buffer. 
Electrophoretic mobility shift assay. FITC-conjugated RNA sequences were incubated with immunoprecipitated CPEB2A-FLAG protein in binding buffer (16) then electrophoresed on a $5 \%$ acrylamide gel in $0.5 \times$ TBE. Fluorescent bands were visualized.

siRNA Transfection. Custom and validated siRNA targeted towards CPEB2B utilized in this study as described previously (16)

Nascent protein labeling. Protocol was adapted from DeLigio et al, 2017 (15). Cells were incubated in RPMI medium with $200 \mu \mathrm{M}$ L-azidohomoalanine for 5 hours. Cells were then harvested as previously described and lysed in lysis buffer (1\% NP-40 in $50 \mathrm{mM}$ Tris- $\mathrm{HCl}, \mathrm{pH}$ 8.0, $1 \times$ protease and phosphatase inhibitors, added fresh). Cells were then sonicated and centrifuged to clear the lysate. Nascent proteins were labeled with biotin using the Click-it protein reaction buffer kit (Thermo Fisher) according to the manufacturer's instructions. Nascent proteins were precipitated using streptavidin-coated magnetic beads. Following 4 washes in PBS $7.4 \mathrm{pH}+/-1 \% \mathrm{NP} 40$, samples were subjected to PAGE-immunoblot.

HUVEC spheroid generation. HUVEC spheroids of defined cell number were generated as previously described $(\mathbf{2 8}, \mathbf{2 9})$.. In brief, HUVECS below passage 7 were grown to $75-90 \%$ confluency and trypsinized. Cells were re-suspended in EGM-2 Lonza medium containing $20 \%$ methylcellulose and cultured as hanging drops (500 cells / $25 \mu \mathrm{L}$ ) onto the lid of a petri dish and $\begin{array}{llllll}\text { incubated at } & 37 & \mathrm{C} & \text { overnight }\end{array}$

In vitro sprouting assay. Approximately 100 HUVEC spheroids were collected by gently washing with $10 \times$ PBS and centrifuging at $500 \mathrm{~g} \times 5 \mathrm{~min}$. On ice, rat-tail collagen was mixed with DMEM (8:1) and $\mathrm{NaOH}$ (enough to change solution from yellow to faint pink). Spheroids were resuspended in EGM-2 Lonza media supplemented with 30\% FBS, and $0.25 \%$ methylcellulose. Immediately following the addition of the collagen:DMEM mixture to the spheroids, 500uL of each spheroid mixture was rapidly plated into a 24-well plate and allowed to polymerize at $37 \mathrm{C}$ for 30 minutes. After polymerization, cells were exposed to hypoxia as described above. Sprout number per spheroid was counted, as well as cumulative sprout length using the measurement tool in FIJI/ImageJ.

Table 1. RNA and DNA Sequences.

\begin{tabular}{|l|l|}
\hline Oligo & Sequence $\left(\mathbf{5}^{\prime} \rightarrow \mathbf{3}^{\prime}\right)$ \\
\hline B-ASO & mGmGmCmCmUUGUAGGUCUUAUCmUmGmGmUmC \\
\hline Control ASO (c-ASO) & mCmCmGmGmAACAUCCAGAAUAGmAmCmCmAmG \\
\hline CPEB2 splicing primers & $\begin{array}{l}\text { F: GCAGCAGAGGAACTCCTATAAC } \\
\text { R: CAAAGAGTGCATATTCAAACTGTCA }\end{array}$ \\
\hline FITC-RNA HIF1 $\alpha$ 3' UTR & Fi: UUAAAAAAAUGCACCUUUUUAUUUAUUUAUUUUUGGCUAG \\
\hline Biotinylated RNA HIF1a 3' UTR & Bi: UUAAAAAAAUGCACCUUUUUAUUUAUUUAUUUUUGGCUAG \\
\hline Biotinylated RNA HIF1a exon 10 & Bi:UAGUCCCAGUGAAUAUUGGUUUUUAUGUGGAUAGUGAUAUGG \\
\hline $\begin{array}{l}\text { Biotinylated RNA HIF1 } \\
\text { (mut) exon } 10\end{array}$ & Bi:UAGUCCCAGUGAAUAUUGGCUUCUACGUGGAUAGUGAUAUGG \\
\hline
\end{tabular}




\section{References Cited}

1. Zimna A, Kurpisz M. Hypoxia-Inducible factor-1 in physiological and pathophysiological angiogenesis: Applications and therapies. BioMed Research International. 2015.

2. Krock BL, Skuli N, Simon MC. Hypoxia-Induced Angiogenesis: Good and Evil. Genes and Cancer. 2011 Dec;2(12):1117-33.

3. Molinari N, Chanez P, Roche N, Ahmed E, Vachier I, Bourdin A. Rising total costs and mortality rates associated with admissions due to COPD exacerbations. Respir Res. 2016. Nov 14;17(1):149.

4. Rello J, Storti E, Belliato M, Serrano R. Clinical phenotypes of SARS-CoV-2: Implications for clinicians and researchers. European Respiratory Journal. 2020. 10.1183/13993003.01028-2020

5. Tursynbek Nurmagambetov, Robin Kuwahara, Paul Garbe. The Economic Burden of Asthma in the United States, 2008 - 2013. Ann Am Thorac Soc. 2018 Mar;15(3):348356.

6. Zhang B, Niu W, Dong HY, Liu ML, Luo Y, Li ZC. Hypoxia induces endothelial-mesenchymal transition in pulmonary vascular remodeling. Int $\mathrm{J}$ Mol Med. 2018 Jul;42(1):270-278.

7. Ball MK, Waypa GB, Mungai PT, Nielsen JM, Czech L, Dudley VJ, Schumacker PT Regulation of hypoxia-induced pulmonary hypertension by vascular smooth muscle hypoxia-inducible factor-1a. Am J Resp Crit Care Med, 2014;189(3), 314-324.

8. Shimoda LA, Manalo DJ, Sham JSK, Semenza GL, Sylvester JT. Partial HIF-1a deficiency impairs pulmonary arterial myocyte electrophysiological responses to hypoxia. Am J Phys Lung Cell Mol Phys; 2001; 281(1):L202-L208.

9. Li, Z., Wang, D., Messing, E. M., \& Wu, G. VHL protein-interacting deubiquitinating enzyme 2 deubiquitinates and stabilizes HIF-1a. 2005 EMBO Reports. 2005 Apr;6(4):373-8.

10. Epstein AC, Gleadle JM, McNeill LA, Hewitson KS, O'Rourke J, Mole DR, Mukherji M, Metzen E, Wilson MI, Dhanda A, et al. C. elegans EGL-9 and mammalian homologs define a family of dioxygenases that regulate HIF by prolyl hydroxylation. Cell. 2001;107:43-54.

11. Kimura H, Weisz A, Ogura T, Hitomi Y, Kurashima Y, Hashimoto K, D'Acquisto F, Makuuchi M, Esumi $\mathrm{H}$. Identification of hypoxia-inducible factor 1 ancillary sequence and its function in vascular endothelial growth factor gene induction by hypoxia and nitric oxide. J Biol Chem. 2001 Jan 19;276(3):2292-8.

12. Pastukh V, Roberts JT, Clark DW, Bardwell GC, Patel M, Al-Mehdi AB, Borchert GM, Gillespie MN. An oxidative DNA "damage" and repair mechanism localized in the VEGF promoter is important for hypoxia-induced VEGF mRNA expression. Am J Physiol Lung Cell Mol Physiol. 2015 Dec 1;309(11):L1367-75.

13. Kimura H, Weisz A, Ogura T, Hitomi Y, Kurashima Y, Hashimoto K, D'Acquisto F, Makuuchi M, Esumi $\mathrm{H}$. Identification of hypoxia-inducible factor 1 ancillary sequence and its function in vascular endothelial growth factor gene induction by hypoxia and nitric oxide. J Biol Chem. 2001 Jan 19;276(3):2292-8. 
14. Apte RS, Chen DS, Ferrara N. VEGF in Signaling and Disease: Beyond Discovery and Development. Cell. 2019 Mar 7;176(6):1248-1264.

15. DeLigio, JT, Lin G, Chalfant, CE, Park MA. Splice variants of cytosolic polyadenylation element- binding protein 2 (CPEB2) differentially regulate pathways linked to cancer metastasis. J Biol Chem. 2017; 292(43), 17909-17918.

16. Johnson RM, Vu NT, Griffin BP, Gentry AE, Archer KJ, Chalfant CE, Park MA. The Alternative Splicing of Cytoplasmic Polyadenylation Element Binding Protein 2 Drives Anoikis Resistance and the Metastasis of Triple Negative Breast Cancer. J Biol Chem. 2015 Oct 16;290(42):25717-27.

17. Deligio, JT, Stevens, SC, Nazario-Muñoz, GS, MacKnight, HP, Doe, KK, Chalfant, CE, \& Park, MA. Serine/arginine-rich splicing factor 3 modulates the alternative splicing of cytoplasmic polyadenylation element binding protein. Mol Cancer Res. 2019 Sep;17(9):1920-1930.

18. Mendez, R., \& Richter, J. D. (2001). Translational control by CPEB: A means to the end. Nature Rev Mol Cell Biol. 2001 Jul;2(7):521-9.

19. Richter JD. CPEB: a life in translation. Trends in Biochem Sci. 2007 Jun;32(6):279-85.

20. Reiterer M, Colaço R, Emrouznejad P, Jensen A, Rundqvist H, Johnson RS, Branco C. Acute and chronic hypoxia differentially predispose lungs for metastases. Sci Rep. 2019; Jul 5; 9(1):10246.

21. Hutt DM, Roth DM, Vignaud H, Cullin C, Bouchecareilh M. The histone deacetylase inhibitor, vorinostat, represses hypoxia inducible factor 1 alpha expression through translational inhibition. PLoS One. 2014; Aug 28;9(8):e106224

22. Hake LE, Mendez R, Richter JD. Specificity of RNA Binding by CPEB: Requirement for RNA Recognition Motifs and a Novel Zinc Finger. Mol Cell Biol. 1998; Feb; 18(2):685-93

23. Huang YS, Kan MC, Lin CL, Richter JD. CPEB3 and CPEB4 in neurons: Analysis of RNA-binding specificity and translational control of AMPA receptor GluR2 mRNA. EMBO J. 2006; Oct 18;25(20):4865-76.

24. Dengler, VL, Galbraith, MD, Espinosa, JM. Transcriptional regulation by hypoxia inducible factors. Crit Rev Biochem Mol Biol. 2014 Jan-Feb;49(1):1-15.

25. Chen, PJ, \& Huang, YS. CPEB2-eEF2 interaction impedes HIF-1a RNA translation. EMBO J. 2012 Feb 15;31(4):959-71.

26. Vu NT, Park MA, Shultz MD, Bulut GB, Ladd AC, and Chalfant CE. Caspase-9b interacts directly with clAP1 to drive agonist-independent activation of NF-kB and lung tumorigenesis. 2016. Cancer Res. 76, 2977-2989

27. Shapiro BA, Vu NT, Shultz MD, Shultz JC, Mietla JA, Gouda MM, Yacoub A, Dent P, Fisher PB, Park MA, and Chalfant CE. Melanoma differentiation-associated gene 7/IL-24 exerts cytotoxic effects by altering the alternative splicing of Bcl-x pre-mRNA via the SRC/PKCס signaling axis. 2016. J. Biol. Chem. 291, 21669-21681

28. Heiss M, Hellström M, Kalén M, May T, Weber H, Hecker M, et al. Endothelial cell spheroids as a versatile tool to study angiogenesis in vitro. FASEB J. 2015 Jul;29(7):3076-84

29. Tetzlaff F, Fischer A. Human Endothelial Cell Spheroid-based Sprouting Angiogenesis Assay in Collagen. BIO-PROTOCOL. 2018. Elife 7: e32860

30. Zimna A, Kurpisz M. Hypoxia-Inducible factor-1 in physiological and pathophysiological angiogenesis: Applications and therapies. BioMed Research International.2015:549412 
31. Kim, J., Park, R. Y., Chen, J. K., Kim, J., Jeong, S., \& Ohn, T. Splicing factor SRSF3 represses the translation of programmed cell death 4 mRNA by associating with the $5^{\prime}$ UTR region. Cell Death and Differentiation. 2014. 21, 481-490

32. Kato M, Han TW, Xie S, Shi K, Du X, Wu LC, Mirzaei H, Goldsmith EJ, Longgood J, Pei J, Grishin NV, Frantz DE, Schneider JW, Chen S, Li L, Sawaya MR, Eisenberg D, Tycko R, McKnight SL. Cell-free formation of RNA granules: low complexity sequence domains form dynamic fibers within hydrogels. Cell. 2012 May 11;149(4):753-67.

33. Apte RS, Chen DS, Ferrara N. VEGF in Signaling and Disease: Beyond Discovery and Development. Cell. 2019 Mar 7;176(6):1248-1264.

34. Hake LE, Mendez R, Richter JD. Specificity of RNA Binding by CPEB: Requirement for RNA Recognition Motifs and a Novel Zinc Finger. Mol Cell Biol. 1998; Feb;18(2):685-93.

35. Dengler, V. L., Galbraith, M. D., \& Espinosa, J. M. (2014). Transcriptional regulation by hypoxia inducible factors. Crit Rev Biochem Mol Biol. 2014 Jan-Feb;49(1):1-15.

36. Hanna J, Leggett DS, Finley D. Ubiquitin depletion as a key mediator of toxicity by translational inhibitors. Mol Cell Biol. 2003 Dec;23(24)3. PMID: 14645527; PMCID: PMC309641. 